\title{
Prevalence of Significant Carotid Stenosis and Other Risk Factors in Patients with Acute Ischemic Stroke in Yaounde, Cameroon
}

\author{
Ngo Nonga $B^{1 *}$, MBalla $\mathrm{JC}^{2}$, Ntone $\mathrm{F}^{3}$, Ndongo $\mathrm{S}^{3}$, Ouankou $\mathrm{C}^{3}$, Handy ED ${ }^{1}$, Omboto $\mathrm{S}^{4}$ and $\mathrm{Ngongang}^{5}$
}

${ }^{1}$ Department of Surgery, University Hospital Centre of Yaounde, Faculty of Medicine and Biomedical Sciences, University of Yaounde I, Yaounde, Cameroon ${ }^{2}$ Department of Radiology, University Hospital Centre of Yaounde, Faculty of Medicine and Pharmacy, University of Douala, Douala, Cameroon

${ }^{3}$ Department of Medicine, University Hospital Centre of Yaounde, Faculty of Medicine and Biomedical Sciences, University of Yaounde I, Yaounde, Cameroon

${ }^{4}$ Department of Biochemistry, University Hospital Centre of Yaounde, Faculty of Medicine and Biomedical Sciences, University of Yaounde I, Yaounde, Cameroon

\begin{abstract}
The prevalence and incidence of cardiovascular disease have been increasing in sub Saharan Africa for the last past twenty years. One of the most common manifestations of cardiovascular disease is stroke. Very few studies have addressed the major causes of ischemic stroke in this region. The objective of this study was to determine the prevalence of significant extra cranial carotid stenosis in a population of patients with acute ischemic stroke.
\end{abstract}

Patients and methods: we conducted a prospective study at the University Hospital Centre of Yaoundé from January 2013 to October 2013. We included all consenting patients with acute onset of neurological deficit with a CT scan of the head showing an ischemic stroke. We excluded all patients with hemorrhagic stroke on CT scan, or who did not have a head CT scan. The study was approved by the National ethics committee.

Results: During that period, 35 patients met the criteria for acute ischemic stroke from which $51.4 \%$ were women with a sex ratio of 0.94 . The mean age in this series was 66.6 years. Thirty five patients over 76 had an ischemic stroke on the CT scan, with a prevalence of $46 \%$. Hypertension was present in $21(60 \%)$ cases and it was a major risk factor. Only 3 patients had a carotid stenosis between $50-75 \%$, there was no patient with a stenosis above $75 \%$. Elevated cholesterol was found in $3.3 \%$ of patients while $30(86 \%)$ patients had an abnormal electrocardiogram: 9 patients were found to have a tachyarrhythmia and 6 atrial fibrillation. There was no intracardiac clot even though the echocardiogram was abnormal in $26(77 \%)$ patients. Concerning the CT scan of the head results, hypo densities were found in $26(74.3 \%)$ patients and the CT was normal in $9(9.9 \%)$ patients with a sudden neurological deficit.

Conclusion: From this pilot study, we have found that carotid stenosis in association with hypercholesterolemia and arteriosclerosis may not be a major risk factor for ischemic stroke in our environment, while age, hypertension and arrhythmia are the most important risk factors for this disease in Cameroon. Further and larger study is needed to confirm this preliminary finding.

Keywords: Stroke; Ischemic; Carotid stenosis; Arrhythmia; Hypertension

\section{Introduction}

Worldwide cardiovascular disease globally represents one of the leading causes of death and its incidence is increasing in sub-Saharan Africa [1]. One of the most common manifestations of cardiovascular disease is stroke. In western countries, ischemic stroke is more common than hemorrhagic stroke; Ischemic stroke has been strongly associated with intracranial occlusive disease in Africans American and less with extra cranial carotid disease while carotid diseases is more prevalent in Caucasian [2,3]. Very few studies have been published on strokes and related risk factors in Cameroon. Hypertension is the most important risk factor for stroke worldwide, In Black American, Gillum have reported that hypertension, diabetes mellitus, age and smoking are the most important risk factors in Africans American Hyperlipidemia is less prevalent in this population [2]. Extra cranial carotid disease due to arteriosclerosis is usually associated with hypercholesterolemia and hyper lipidemia [1]. Extra cranial carotid stenosis has been found to be less prevalent in black American and in Egyptians while intracranial disease is far more common [4,5], But few studies have been published on the subject and none has addressed the issue of extra cranial or intracranial stenosis and other commonly found risk factors in stroke in Cameroon.

The objective of this prospective study was to determine the prevalence of significant extra cranial carotid stenosis and others risks factors of ischemic stroke in Yaoundé, Cameroon.

\section{Patients and Methods}

We conducted a single institution prospective study from January 2013 to October 2013. Were included in the study all consenting patients admitted in the emergency service of the university teaching hospital of Yaounde for ischemic stroke. The stroke was defined as sudden onset of neurological deficit with or without loss of consciousness and classified on the CT scan as hemorrhagic or ischemic type (either an image on the $\mathrm{CT}$, or acute neurological deficit with a normal scan). Ischemic stroke was considered in patients presenting a sudden onset of a neurological deficit and a CT scan image of ischemia (hypo densities) or a normal CT scan.

Were excluded patients with a progressive neurological deficit,

*Corresponding author: Ngo Nonga B, Attending Surgeon, Associate Professor of Surgery, University Hospital Centre of Yaounde, Yaounde, Cameroon, Tel: 00237699866422; E-mail: ngonongab@yahoo.com

Received February 22, 2016; Accepted March 01, 2016; Published March 09, 2016

Citation: Ngo Nonga B, MBalla JC, Ntone F, Ndongo S, Ouankou C, et al. (2016) Prevalence of Significant Carotid Stenosis and Other Risk Factors in Patients with Acute Ischemic Stroke in Yaounde, Cameroon. J Vasc Med Surg 4: 257. doi:10.4172/2329-6925.1000257

Copyright: (c) 2016 Ngo Nonga B, et al. This is an open-access article distributed under the terms of the Creative Commons Attribution License, which permits unrestricted use, distribution, and reproduction in any medium, provided the original author and source are credited. 
children below 16 years, hemorrhagic strokes, non-consenting patient, acute neurological deficit with no CT scan, and patients without CT scan results?

Data were prospectively collected and stored in a special file containing numerous variables taken from the medical records: charts were reviewed for risk factors of stroke: age, sex, past medical history, (arrhythmia, hypertension, diabetes mellitus, stroke, heart disease). Hypertension was considered when the blood pressure was more than 140/90.

Systematic electrocardiogram (EKG), echocardiogram, extra cranial carotid duplex ultrasound, and a lipid profile were realized in all patients with ischemic stroke to look for risk factors and etiological factors. The reading of the EKG and cardiac ultrasound were done by two cardiologists who reviewed and discussed all the exams. The carotid duplex ultrasound was done by the same radiologist. The lipid profile was done by the same laboratory. Carotid arteriosclerosis was assessed clinically (murmur) and by duplex ultrasound. The stenosis was found to be significant if it was above $50 \%$, and hemodynamically significant if the stenosis occupied $3 / 4$ of the circumference of the carotid. The cut off for Hypercholesterolemia was considered if the number was more than $2,7 \mathrm{~g} / \mathrm{l}$, and LDL cholesterol more than $1,88 \mathrm{~g} / \mathrm{l}$. triglycerides 1,5 $\mathrm{g} / \mathrm{l}$, HDL below $0,44 \mathrm{~g} / \mathrm{l}$. The echocardiogram and the carotid duplex ultrasound were done using the same machine (DC N3, made by MINDRAY from CHINA).

The statistical analysis was done using the SPSS version 16.0 for windows.

The study was approved by the hospital internal review board, the faculty of medicine ethical committee and the National ethic committee of Cameroon.

\section{Results}

During the ten months period, 83 patients were admitted thru the emergency room with the diagnosis of acute stroke. Seventy six of these patients had a CT scan of the head done. Hemorrhagic stroke was found in 41 of the CT scan, while Ischemic stroke was found in 35 of them giving prevalence among stroke of $46 \%$.

More than half of the patients with ischemic stroke (51.4\%) were women with a sex ratio of 0.94 . The mean age was 66.6 years with a minimum of 41 and maximum of 90 years, the standard deviation was 13.86 years the mode was around 70 years, $34.5 \%$ of the patients were between 70 and 79 years. For age in between 50-59, men were more present, and for age in between 70-79, women were more prevalent (44\%). There was no patient below 40 years, making Age be considered as a risk factor.

Only 12 patients (34.2\%) came to the hospital the day of the onset of symptoms, 8 (22.8\%) patients came the second day after the onset of the neurological deficit, and 15 patients (42.8\%) consulted after 48 hours, the mean time for the delay for consultation was 2.66 days.

Twenty one patients (60\%) were found to have hypertension, from them 9 were men and 12 were women. While 14 (40\%) patients did not have hypertension, 8 were men and 6 were women. The difference between the hypertension and the non-hypertension group was not significant; $p=0.24$. No patient in this group had a history of tobacco abuse.

Physical finding are shown in Table 1. Right hemiplegia or hemiparesia were found in 19 patients (54.3\%), left hemiplegia or hemiparesia in 12 patients (33.9\%), Loss of consciousness 11 (31.5\%) patients. The Glasgow coma scale ranged from 5 (1 patient) to 15.

Only three $(8.6 \%)$ patients were found to have a carotid murmur More than $2 / 3$ of the patients $(68.5 \%)$ had a hemiplegia on admission. Only one patient was diabetic. The other 35 patients had a normal blood sugar and had no history of elevated blood sugar.

The CT scan was requested on arrival to the hospital, but there was a delay for its realization. Only 7 (20\%) had it done in the first 24 hours, 12 the second day of admission (3.2\%) between 24 and 48 hours, and $16(45.7 \%)$ after 48 hours. The hypo densities images were found in patients with ischemic stroke when the scan was done after 48 hours. Nine patients had a normal CT scan with a clinical neurological deficit from which, 3 scan were done the same day of admission and 6 the next day of admission. All the scans (45\%) done after the second day of admission were positive and showed hypo densities.

From the patients with hypo densities on the CT scan done after 48 hours, 17 had hypertension, and 9 had normal blood pressure. Most of the hypo densities were localized in the parietal region involving the middle cerebral artery (13 patients), from which 6 had hypertension. Five patients had a hypo density in the parieto-frontal region, they were all hypertensive and the corresponding vascular territory was at the junction of the anterior cerebral artery and middle cerebral artery. The difference between the hypertension group and the non hypertension group for this localization was significant with a $\mathrm{p}=0.049$; CI $95 \%$ (1.34-2.62). Four patients had an ischemic stroke involving the junction between the middle cerebral artery and the posterior cerebral artery; only two patient's lesion involved the anterior cerebral artery and the posterior cerebral artery each.

\section{Carotid duplex ultrasound}

17 patients had an abnormal carotid ultrasound, from which 14 had carotid irregularities and non-ulcerated atheroma with no significant stenosis, 3 presented a stenosis between $50-75 \%$, 2 lesions were on the right and one lesion on the left, there was no lesion above $75 \%$. From the patients with carotid abnormalities, 15 had hypertension and 2 only did not have hypertension one of them had a stenosis between $50-75 \%$. Hypertension was associated with the presence of a positive carotid duplex ultrasound with an OR of 2.160 CI 95\% (1.46-3.53); $\mathrm{p}=0.002$.

Lipid Profile: a lipid profile was done in 31 patients (88.6\%), only $3.3 \%$ had an elevated total cholesterol and LDL cholesterol. All the patients had normal triglyceride and HDL cholesterol. The rest of the laboratory results did not show any significant abnormalities.

\section{Electrocardiogram (EKG)}

$30(86 \%)$ patients had an abnormal EKG; (abnormal EKG was

\begin{tabular}{|c|c|}
\hline Signs and symptoms & $\mathbf{N}(\%)$ \\
\hline Headache & $14(11.5$ \\
\hline vertigo & $3(8.6 \%)$ \\
\hline Memory lost & $3(8.6 \%)$ \\
\hline Convulsions & $3(8.6 \%)$ \\
\hline Falcial palsy & $10(28.6 \%)$ \\
\hline hemiplegia & $24(68.5 \%)$ \\
\hline hemiparesia & $7(20 \%)$ \\
\hline aphasy & $4(11.5 \%)$ \\
\hline Carotid murmur & $3(8.6 \%)$ \\
\hline Inconsciousness & $11(31.4 \%)$ \\
\hline
\end{tabular}

Table 1: Signs and symptoms of the patients on admission. 


\begin{tabular}{|c|c|c|c|c|}
\hline Type of exam and its results & Group I: Elevated BP & Group II: Normal BP & P-value & $\mathrm{Cl} 195 \%$ \\
\hline \multicolumn{5}{|c|}{ EKG results } \\
\hline LVH & 7 & 4 & 0.70 & {$[0.28-5.44]$} \\
\hline tachyarrhythmia & 5 & 4 & 0.75 & [0.16-3.62] \\
\hline Atrial fibrillation & 3 & 3 & 0.5 & {$[0.10-3.6]$} \\
\hline Heart block & 1 & 1 & 0.77 & [0.037-11.33] \\
\hline Sinus bradycardia & 1 & 1 & 0.32 & {$[0.025-3.67]$} \\
\hline Total & 17 & 13 & & \\
\hline \multicolumn{5}{|c|}{ Echocardiogram } \\
\hline Diastolic dysfunction & 1 & 0 & 0.40 & {$[1.28-2.25]$} \\
\hline Systolic dysfunction & 1 & 2 & 0.32 & [0.025-3.67] \\
\hline Valvular calcification & 2 & 0 & 0.2 & [1.29-2.32] \\
\hline LVH & 4 & 4 & 0.51 & {$[0.12-2.88]$} \\
\hline LAH & 7 & 5 & 0.88 & {$[0.21-3.7]$} \\
\hline Total & 15 & 11 & & \\
\hline \multicolumn{5}{|c|}{ Carotid duplex ultrasound } \\
\hline Irregularities without stenosis & 13 & 1 & 0.002 & {$[1.46-3.53]$} \\
\hline Carotid stenosis $50-75 \%$ & 2 & 1 & 0.80 & {$[0.11-16.7$} \\
\hline Total & 15 & 2 & & \\
\hline \multicolumn{5}{|c|}{ CT scan of the head results } \\
\hline Hypo densities & 17 & 9 & & \\
\hline Normal, but neurological deficit & 4 & 5 & & \\
\hline
\end{tabular}

Table 2: Comparison of the results of the electrocardiogram, echocardiogram, carotid duplex ultrasound and CT scan of the head in both group.

found in 17 of the 21 patients with hypertension, and in 13 of the 14 patients without hypertension. The results of the EKG are summarized in Table 2. Left ventricular hypertrophy followed by tachyarrhythmia was the most common lesion. Left ventricular hypertrophy was also found in non-hypertensive patients, making us believe that they may suffer from a paroxysmal rise of the blood pressure.

\section{Cardiac ultrasound}

26 patients were able to do the exam; 15 had an elevated blood pressure, and 11 had a normal blood pressure. Atrial hypertrophy was present in both groups (Table 2). Four patients in the no-hypertension group presented left ventricular hypertrophy, making us believe that these patients may have been suffering from hypertension too. There was no intracardiac thrombus or clot.

Most of the patients were discharged home after 2 days, patients with low Glasgow coma score and those who did the CT scan with a delay stay longer than usual. One patient with a multifocal ischemic stroke died few days after admission.

\section{Discussion}

The prevalence of ischemic stroke subtype in this study is $46 \%$. This is lower than the reported $85 \%$ prevalence rate in one study in Kenya [6] and 77.6\% reported in one study in Kampala [7]. Ischemic stroke is also the most common type of stroke in western countries [2]. The mean age is similar to one found in the Kenyan study [6] 68 years compared to 66.6 years in our study. As reported in many studies, age is risk factors for ischemic stroke in our patients and it is lightly more prevalent in women than in men [1-3].

In this study, Hypertension was found to be a very common risk factor for ischemic stroke mainly located in the front-parietal region depending on the territory of the anterior and middle cerebral artery.

Close to $86 \%$ of our patients have an arrhythmia with atrial fibrillation and tachyarrhythmia being present in 15 (50\%) of them. Although there was no intracardiac clot on the regular cardiac sonogram, it is possible that the ischemia be due to emboli originating from the heart. It would have been good to do a transesophageal echocardiogram to be able to rule out intracardiac clots. This test is not being currently done in our hospital and in Cameroon as a whole. Many patients with no history of hypertension were having left ventricular hypertrophy on the EKG and cardiac sonogram making us believe that they may have presented paroxysmal hypertension. Considering this make hypertension as the most important risk factor for ischemic stroke in our serie as it has also been reported $[1,2,6]$. Nevertheless, from our study we can say that hypertension and arrhythmia are creating a favorable environment for stroke. This finding has been reported by Jowi et al. in Kenya [6]. On the other hand EKG abnormalities were very common and Joubert et al. have reported an elevated prevalence of ischemic heart disease in stroke patients in South Africa [8]. We have not look for specifically ischemic heart disease, but abnormalities in the EKG are in favor of some kind of common cardiac diseases in our patients. Diabetes Mellitus was less present in our patients as one may expected, we have no explanation and this point may need further studies with a greater number of patients.

Very few $(3.3 \%)$ of patients in this series has been found to have elevated blood cholesterol (total or LDL), HDL-cholesterol was not also elevated. We have not tested for homo cystinemia. The absence of elevated LDL and total cholesterol may explain the low incidence of a significant carotid arteriosclerotic disease. The abnormalities found were due to advanced age.

Although malaria is endemic in Cameroon, there was no stroke related to malaria as David Celemerger et al. have reported [9].

Considering the carotid duplex ultrasound, clinically significant extra cranial carotid disease is uncommon in ischemic stroke in our study along with small number of patients with abnormal cholesterol. Some authors have reported the high prevalence of intracranial disease compared to extra cranial in African patients as in Africans American. Due to unavailability, we were unable to assess either with a MRI or an angiogram, the presence or absence of intracranial disease [3]. This 
low incidence of significant carotid stenosis in Blacks patients has been reported by others authors [2-4].

Others common known factors like diabetes Mellitus, smoking and alcohol abuse were found in our study. The prevalence and incidence of smoking in the general population has not been well characterized as it is difficult to conduct large epidemiological and reliable studies in Cameroon.

The most common risk factors for ischemic stroke found in our study were older age and hypertension. Age and hypertension which are worldwide well known risk factors. The third may be arrhythmia as the majority of the patients had some kind of arrhythmia. Most of our patients had a normal lipid profiles and very few had arteriosclerosis of the extra cranial carotid vessels. The major difference between this study and other are the absence of arteriosclerosis, low prevalence of ischemic stroke compare to hemorrhagic stroke, very few diabetic cases in the ischemic stroke group. Preventing heart clot and treating hypertension may be the most important means to prevent recurrent stroke in our environment.

\section{Conclusion}

From this preliminary study, hemorrhagic stroke is more prevalent in Cameroon than ischemic stroke and Age with hypertension are the most important risk factors for ischemic stroke. Another very important factor is cardiac arrhythmia and abnormalities. The low prevalence of cholesterol abnormalities and the low prevalence of tobacco abuse associated with a lower occurrence of arteriosclerosis may be related to the low incidence of a significant carotid stenosis. As such endarterectomy is not the most useful measures to prevent ischemic stroke in Cameroon at present. Further studies in a large number of patients are necessary to better assess the prevalence of the lipid abnormalities in a sub-Saharan country with a changing diet associated to the dosage of homocystinemia.

\section{References}

1. Celermajer DS, Chow CK, Marijon E, Anstey NM, Woo KS (2012) Cardiovascular disease in the developing world: prevalences, pattern and the potential of early disease detection. Journal of the American College of Cardiology 60: 12071216.

2. Gillum RF (1999) Risk factors for stroke in blacks: a critical review. Am J Epidemiol 150: 1266-1274.

3. Rajamani K, Sunbuli M, Jacobs BS, berlow E, Marsh JD et al. (2006) Detection of carotid stenosis in Africans American with ischemic heart disease. Journal of Vascular Surgery 43: 1162-1165.

4. Moustafa RR, Moneinm AA, Salem HH, Shalash AS, Azmy HA (2013) Intracranial-steno-occlusive arterial disease and its association in Egyptian ischemic stroke patients. Stroke 44: 538-541.

5. Ahuja RM, Chaturvedi S, Eliott D, Joshi N, Puklin JE et al. (1999) Mechanisms of retinal arterial occlusive disease in Africans American and Caucasian patients. Stroke 30: 1506-1509.

6. Jowi JO, Mativo PM (2008) Pathological sub-types, risk factors and outcome of stroke at the Nairobi Hospital, Kenya. East Afr Med J 85: 572-581.

7. Jane N, Nyakoojo A, Alice N, Nicolas R, Edward D (2012) A descriptive epidemiological study on stroke in Kampala, Uganda: A hospital based- study. African Journal of Neurological sciences. 31: 41-48.

8. Joubert J, McLean CA, Reid CM, Davel D, Pilloy W, et al. (2000) Ischemic heart disease in black South African stroke patients. Stroke 31: 1294-1298.

9. Mohammad Y, Quattan M, Prabhakaran S (2010) Epidemiology and pathophysiology of intracranial large artery stenosis. The Open Arteriosclerosis and Thrombosis Journal 3: 3-7. 